\title{
Molecular imaging of fibrosis: recent advances and future directions
}

\author{
Sydney B. Montesi, ${ }^{1}$ Pauline Désogère, ${ }^{2,3,4}$ Bryan C. Fuchs, ${ }^{5}$ and Peter Caravan ${ }^{2,3,4}$ \\ 'Division of Pulmonary and Critical Care Medicine and ${ }^{2}$ Department of Radiology, Massachusetts Ceneral Hospital and Harvard Medical School, Boston, Massachusetts, USA. ${ }^{3}$ Athinoula A. Martinos Center \\ for Biomedical Imaging and ${ }^{4}$ Institute for Innovation in Imaging, Massachusetts Ceneral Hospital, Boston, Massachusetts, USA. ${ }^{5}$ Division of Surgical Oncology, Massachusetts Ceneral Hospital Cancer Center \\ and Harvard Medical School, Boston, Massachusetts, USA.
}

\begin{abstract}
Fibrosis, the progressive accumulation of connective tissue that occurs in response to injury, causes irreparable organ damage and may result in organ failure. The few available antifibrotic treatments modify the rate of fibrosis progression, but there are no available treatments to reverse established fibrosis. Thus, more effective therapies are urgently needed. Molecular imaging is a promising biomedical methodology that enables noninvasive visualization of cellular and subcellular processes. It provides a unique means to monitor and quantify dysregulated molecular fibrotic pathways in a noninvasive manner. Molecular imaging could be used for early detection, disease staging, and prognostication, as well as for assessing disease activity and treatment response. As fibrotic diseases are often molecularly heterogeneous, molecular imaging of a specific pathway could be used for patient stratification and cohort enrichment with the goal of improving clinical trial design and feasibility and increasing the ability to detect a definitive outcome for new therapies. Here we review currently available molecular imaging probes for detecting fibrosis and fibrogenesis, the active formation of new fibrous tissue, and their application to models of fibrosis across organ systems and fibrotic processes. We provide our opinion as to the potential roles of molecular imaging in human fibrotic diseases.
\end{abstract}

\section{Introduction}

Fibrosis is a progressive scarring process arising from tissue damage or inflammation that can result in both organ damage and failure (1). Fibrotic diseases are responsible for a large proportion of human illness and are estimated to cause almost half of all human deaths in the United States (2). Atrial fibrillation, atherosclerosis, hypertrophic cardiomyopathy, nonalcoholic steatohepatitis (NASH), diabetic nephropathy, idiopathic pulmonary fibrosis (IPF), inflammatory bowel disease, scleroderma, and pancreatic cancer are all examples of major illnesses with a fibroproliferative component (3-5). Despite major advances in our understanding of these fibrotic diseases, there are no currently available treatments to halt fibrogenesis, the active development of new scar formation, or reverse established fibrosis.

The few antifibrotic treatments available at best modify the rate of fibrosis progression $(6,7)$. Fibrotic diseases represent challenging targets for the development of new therapies for multiple reasons. Disease progression can be slow, e.g., in NASH, resulting in long and costly clinical trials. The patient population may be het-

\footnotetext{
Conflict of interest: BCF receives research support from Collagen Medical, Enanta Pharmaceuticals, and Blade Therapeutics. He is a paid consultant for Gilead Sciences. PC has greater than $5 \%$ equity in Collagen Medical, Factor 1A LLC, and Reveal Pharmaceuticals. He receives research support from Pfizer, Pliant Therapeutics, and Indalo Therapeutics, and has been a consultant to Collagen Medical, Guerbet, and Bayer. He is a named inventor on issued or pending patents US7,927,581 (EP-2104R), US8,034,898 (EP-3533, CM-101), US2017360967 (68Ga-CBP8), and W02015085005 (Gd-Hyd, Gd-OA). PD is a named inventor on patent application US2017360967 (68Ga-CBP8).

Reference information: / Clin Invest. 2019;129(1):24-33.

https://doi.org/10.1172/JCl122132.
}

erogeneous and hard to phenotype, e.g., in IPF, making clinical trial data interpretation difficult when slow progressors are enrolled alongside fast progressors. For novel therapeutic targets, evaluation of target expression (is there increased expression of the therapeutic target in diseased tissue?) and target engagement (does the drug engage the target of interest?) in the intended patient population is paramount. In most cases, methods to better stratify patients, quantify target expression or target engagement, and monitor early treatment response within fibrotic diseases are lacking.

Clinical imaging techniques, such as computed tomography (CT), ultrasound, and magnetic resonance imaging (MRI), can detect established fibrosis in the lung, liver, and heart but are less sensitive at detecting early-stage disease, and cannot distinguish active disease (fibrogenesis) from stable scar. As effective treatments become available, earlier detection strategies are needed to enable earlier interventions. There likely also exists significant molecular heterogeneity, differences in the molecular pathways activated within specific fibrotic entities and within different individuals affected by the same fibrotic diseases, that has yet to be elucidated. Such knowledge may allow for targeted therapies to be developed and used. Biopsy is an imperfect strategy for understanding activated molecular pathways and is not without risks.

Molecular imaging emerges as a method to potentially address these important challenges in a safe and noninvasive manner (8, 9). Molecular probes are small molecules, peptides, or antibodies that recognize a specific protein, receptor, or biological process and are tagged with an imaging reporter for visualization. Most imaging modalities can utilize molecular probes, and there is no ideal modality. Positron emission tomography (PET) and single-photon 
A Target engagement

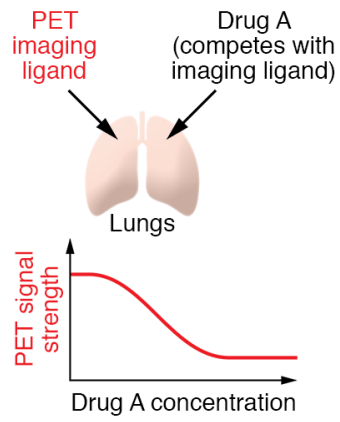

B Target expression

Patient A Patient B

CT

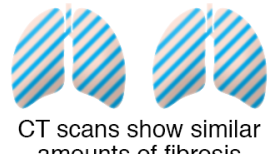

PET

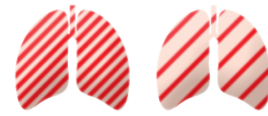

PET scans reveal dissimilar levels of molecular target
C Diagnosis and staging
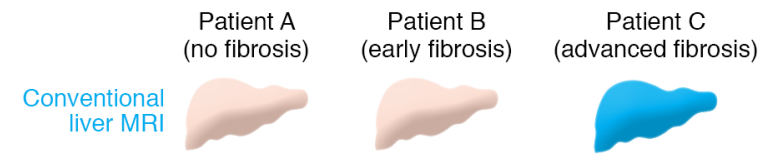

MRI fails to recognize disease stage

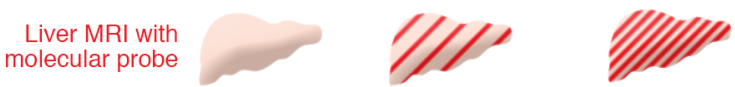

Molecular MRI reveals disease stage
D Cohort enrichment for clinical trials

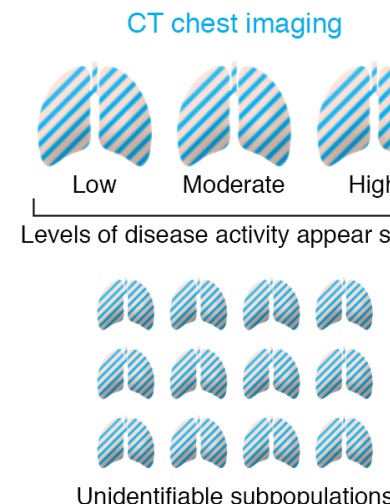

of heterogeneous cohort
PET imaging with molecular probe

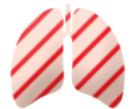

Low disease activity

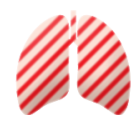

Moderate disease activity

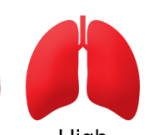

High disease activity

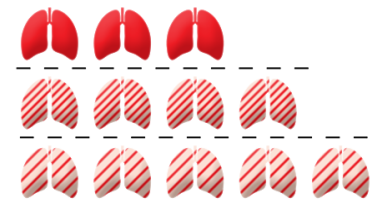

Identifiable subpopulations of heterogeneous cohort
E Treatment response

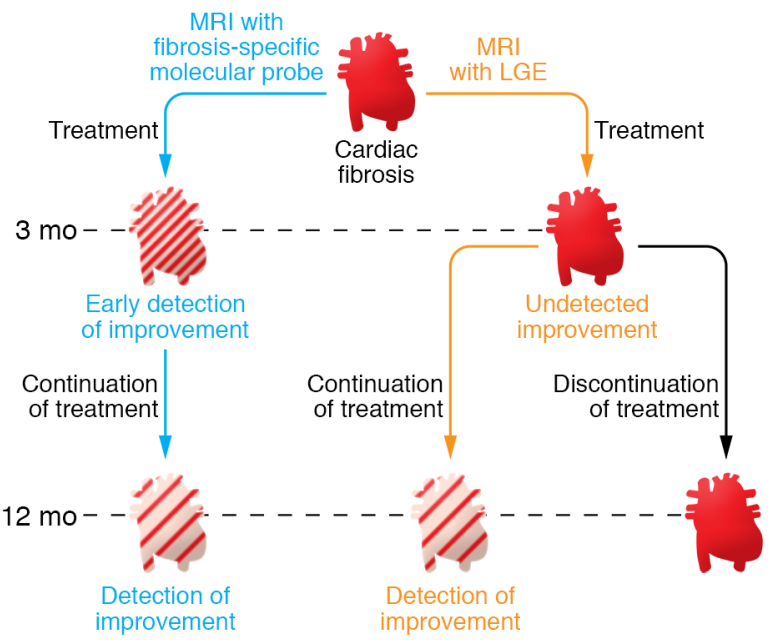

Figure 1. Conceptual applications of molecular imaging in human fibrotic diseases. (A) Target engagement. PET imaging in an early stage clinical trial for a novel antifibrotic therapy. PET ligand binds to the molecular target of Drug A. Modeling the Drug A dose-dependent change in PET signal provides target concentration and affinity of Drug A for the target. (B) Target expression. PET imaging with a molecular probe that binds to a molecular target implicated in pulmonary fibrosis pathogenesis. PET imaging differentiates high versus low expression of the molecular target, selecting patients for treatment with an inhibitor of the molecular target. (C) Diagnosis and staging. Patients at risk for liver fibrosis undergo conventional liver MRI and liver MRI with a molecular probe. Degree of MRI signal enhancement enables earlier detection of fibrosis and noninvasive determination of disease stage. (D) Cohort enrichment for clinical trials. PET imaging with a molecular probe performed on IPF subjects for noninvasive detection of disease activity. Conventional CT demonstrates degree of fibrosis but does not inform as to disease activity. PET signal uptake differentiates subjects by degree of disease activity. This information can be utilized in clinical trials to enrich for subjects most likely to meet prespecified primary endpoints. (E) Treatment response. Subjects with cardiac fibrosis undergo treatment with a novel therapy that reverses fibrosis. MRI using a fibrosis-specific Gd probe detects regression in fibrosis earlier than late gadolinium-enhanced MRI. Note: these are hypothetical scenarios that have not yet been performed in humans.

emission computed tomography (SPECT) can detect picomolar concentrations, bringing most biological targets into detection range, and PET can provide absolute quantification. The sensitivity and quantitation of PET make it well suited to measuring target engagement. However, PET and SPECT have lower spatial resolution than other modalities, require daily probe production, and involve ionizing radiation, which makes these techniques less suitable for following patients over time. MRI provides much higher resolution, no radiation, and additional anatomic and functional imaging, but is more limited in the range of molecular targets that can be detected. Ultrasound contrast agents are limited to the vascular space, while optical imaging requires light access to the tissue, limiting it to superficial or semi-invasive applications. All of these techniques can be made quantitative (10). Modalities can also be combined to provide additional information.
Particularly promising in the application of molecular imaging to fibrosis is that the molecular pathways involved in fibrogenesis span multiple fibrotic disease processes (11). Thus, validated probes for assessing one fibrotic disease, e.g., cardiac fibrosis, may have the potential to assess others, e.g., hepatic fibrosis. Here we review available molecular probes to image pathways involved in fibrosis, their application to different fibrotic processes, the role of molecular imaging in answering unmet challenges in fibrosis, and the next steps needed to translate molecular imaging into human fibrotic diseases.

\section{Molecular imaging in human diseases}

Molecular imaging is invaluable in a number of diseases. In oncology, for example, ${ }^{18} \mathrm{~F}$-fluorodeoxyglucose PET-CT is standard of care for diagnosis, staging, and determination of treatment response for many cancers. In cardiology, SPECT radionuclide 
A

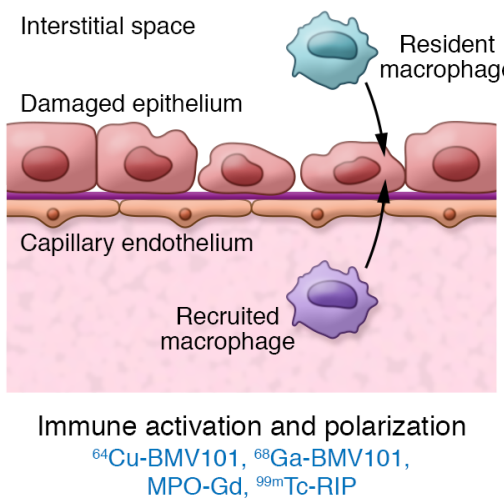

B

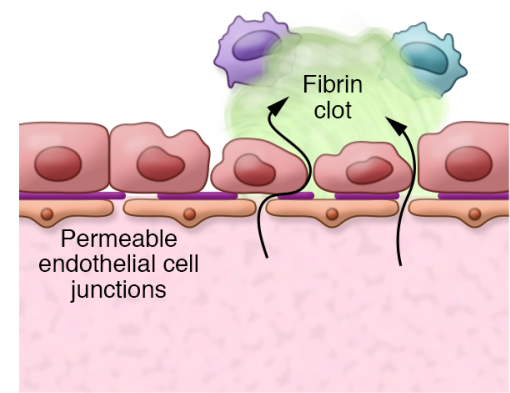

Vascular leak and extravascular coagulation Gadofosveset, EP-2104R, Gd-P, ${ }^{11} \mathrm{C}-\mathrm{BMT}-126088$

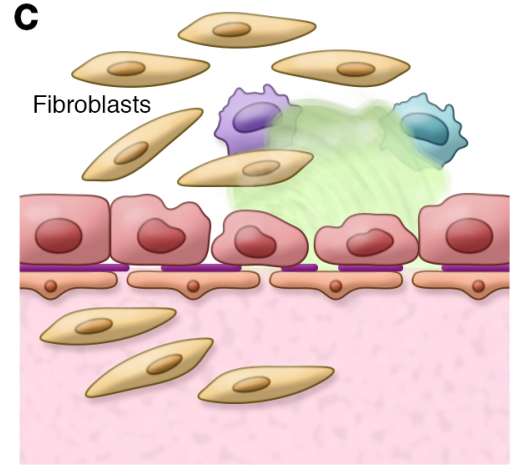

Fibroblast recruitment, invasion, proliferation, and persistence ${ }^{11} \mathrm{C}-\mathrm{BMT}-126088$
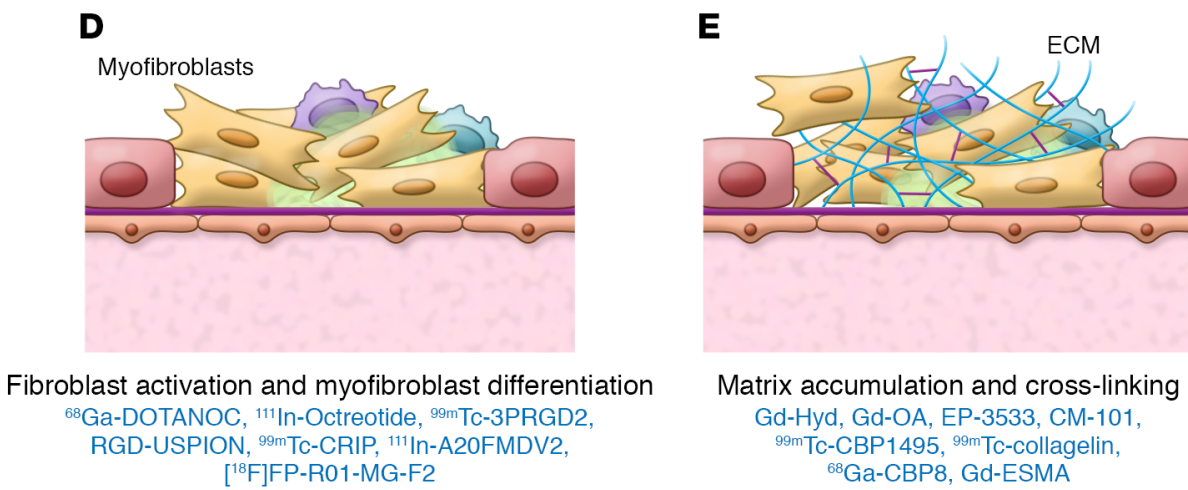

Figure 2. Schematic representation of wound-healing responses resulting in fibrosis. (A) Tissue injury occurs, resulting in cell death and influx of immune cells. Resident and recruited macrophages migrate to the area of injury. (B) Tissue injury also results in increased endothelial permeability (i.e., vascular leak), and activation of the coagulation cascade with formation of a fibrin clot. (C) Fibroblasts migrate to the area of injury. (D) Recruited fibroblasts become activated and differentiate into myofibroblasts. (E) Formation of a provisional extracellular matrix develops and cross-linking occurs. In the setting of normal wound healing, tissue regeneration occurs. During fibrosis, excessive matrix accumulation occurs instead, resulting in organ damage. Available molecular probes are noted by the specific wound-healing response they target or for LPA and $\alpha v \beta 6$, the wound-healing responses they activate. Some probes target or activate more than one wound-healing process. Adapted with permission from the American Journal of Respiratory and Critical Care Medicine (ref. 12), copyright 2018, American Thoracic Society.

imaging can assess cardiac perfusion and viability. In neurology, molecular imaging techniques may assist with earlier diagnosis of neurodegenerative conditions such as Parkinson's disease. While molecular imaging already has an important clinical applicability in many human diseases, to date it has been sparingly applied to fibrotic diseases. With the recent development of molecular probes that target fibrosis-specific or fibrosis-associated processes, molecular imaging is poised to enter the realm of fibrotic diseases. In this context, molecular imaging may enable earlier diagnosis, allow accurate staging of disease, improve phenotyping of those with active disease, select patients for personalized treatment, determine whether a drug is engaging its target, and provide earlier assessments of treatment responses (Figure 1).

\section{Molecular probes for imaging fibrosis}

Fibrosis is the end result of multiple wound-healing processes gone astray (12). While the etiology of the initial tissue insult(s) may be quite varied, from radiation to infection to infarction, the responses to tissue injury are nearly identical regardless of incit- ing injury. At any one point in time, the responses to tissue injury can become dysregulated, shifting the balance from repair and regeneration to fibrogenesis and resultant fibrosis. Many of these processes can be imaged by targeted molecular probes (Figure 2). To date, more than 25 available molecular probes, the majority of which are in the preclinical stage of development, are available to perform molecular imaging of fibrosis and fibrosis-associated processes, such as vascular leak, coagulation, and immune system activation, as we will discuss here (Table 1).

The ideal fibrosis molecular probe should target a molecular process that is specific to fibrosis. The target should be highly expressed in fibrotic tissue and be present at much lower levels elsewhere. Background signal should be low, and this is achieved through low nonspecific binding, rapid elimination, and low off-target uptake. For quantification and disease staging, the uptake (signal) should increase linearly and steeply with increasing disease to provide a large dynamic range. For staging, the precision of the measurement must be high enough that signal changes associated with different stages of disease can be accurately 
Table 1. Molecular probes for fibrosis-specific and fibrosis-associated processes

\begin{tabular}{|c|c|c|c|c|c|}
\hline Probe & Molecular process & Molecular/cell target & Stage of development & Imaging type & Potential clinical uses \\
\hline${ }^{68} \mathrm{Ca}$-DOTANOC ${ }^{\mathrm{A}}$ & Activated fibroblasts & Somatostatin receptor & Human studies & PET & Disease activity \\
\hline${ }^{111}$ In-octreotide & Activated fibroblasts & Somatostatin receptor & FDA approved & SPECT & Disease activity \\
\hline 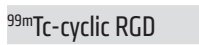 & $\alpha_{4} \beta_{3}$ expression & $\alpha_{4} \beta_{3}$ & Animal studies & SPECT & Disease activity \\
\hline 99mTc-CRIP & $\alpha \beta_{3}$ expression & $\alpha \beta_{3}$ & Animal, human studies & SPECT & Disease activity \\
\hline EP-3533 & Collagen deposition & Type I collagen & Animal studies & MRI & Diagnosis, disease activity, treatment response \\
\hline CM-101 & Collagen deposition & Type I collagen & Animal studies & MRI & Diagnosis, disease activity, treatment response \\
\hline${ }^{99 \mathrm{~m} T \mathrm{~T}-\text {-collagelin }}$ & Collagen deposition & Type I and III collagen & Animal studies & SPECT & Diagnosis, disease activity, treatment response \\
\hline 99mTc-CBP1495 & Collagen deposition & Type I collagen & Animal studies & SPECT & Diagnosis, disease activity, treatment response \\
\hline Gd-ESMA & Elastin deposition & Elastin & Animal studies & MRI & Diagnosis, disease activity, treatment response \\
\hline Gd-Hyd & Lysyl oxidase activity & Allysine, oxidized collagen & Animal studies & MRI & Disease activity, treatment response \\
\hline Gd-OA & Lysyl oxidase activity & Allysine, oxidized collagen & Animal studies & MRI & Disease activity, treatment response \\
\hline${ }^{68} \mathrm{Ca}-\mathrm{BMV101}$ & Activated macrophages & Cysteine cathepsins & Human studies & PET & Disease activity \\
\hline MPO-Gd & Neutrophil degranulation & Myeloperoxidase & Animal studies & MRI & Disease activity, treatment response \\
\hline${ }^{111}$ In-A2OFMDV2 & $\alpha \beta_{6}$ expression & $\alpha \beta_{6}$ & Animal, human studies & PET, SPECT & Disease activity, target expression, treatment response \\
\hline [18 F]FP-R01-MG-F2 & $\alpha_{v} \beta_{6}$ expression & $\alpha_{v} \beta_{6}$ & Human studies & PET & Disease activity, target expression, treatment response \\
\hline "C-BMT-136088 & LPA expression & $\mathrm{LPA}_{1}$ receptor & Animal studies & PET & Target expression, treatment response \\
\hline
\end{tabular}

${ }^{A} \mathrm{~A}$ similar compound, ${ }^{68} \mathrm{Ca}$-DOTATATE, is FDA-approved for imaging of neuroendocrine tumors.

quantified. To measure target engagement, probe uptake should be blockable in a dose-dependent manner by a competing ligand. Not all of the probes described below meet all of these requirements. In some cases the target is not specific to fibrosis (scar), but may still provide useful information about disease activity or the pathogenesis. Since molecular probes are themselves treated as drugs, there is a large regulatory barrier to testing new probes in humans. Thus there is often an application of existing clinical probes for evaluation in fibrosis, e.g., the $\alpha_{\mathrm{v}} \beta_{3}$ and somatostatin receptor probes that were developed for oncology applications.

\section{Fibroblast activation and myofibroblast differentiation}

In response to tissue injury, fibroblasts produce extracellular matrix (ECM) and contribute to formation of granulation tissue, the connective and vascular tissue on the surface of a healing wound (13). During fibrosis, fibroblasts become activated, proliferate, and differentiate into myofibroblasts (14). Somatostatin receptors expressed on normal fibroblasts $(15,16)$ become upregulated in fibrosis (17). ${ }^{111}$ In-octreotide scintigraphy, currently available as the FDA-approved Octreoscan, uses a somatostatin peptide mimic derivatized with a chelated gamma ray-emitting ${ }^{111}$ In isotope. ${ }^{111} \mathrm{In}$-octreotide scintigraphy was performed in subjects with IPF, pulmonary fibrosis due to systemic sclerosis (SSc), and control subjects $(18,19)$. Octreotide expression was increased in IPF and SSc compared with control subjects, with expression higher in IPF than in SSc subjects. ${ }^{111}$ In-octreotide scintigraphy has also been performed on subjects with sarcoidosis and idiopathic interstitial pneumonia. Octreotide uptake was highest in sarcoidosis subjects. IPF subjects in this study, however, had a normal uptake index $(18,19)$. Somatostatin analogs have also been derivatized with PET reporters (20), including the recently FDAapproved ${ }^{68} \mathrm{Ga}$-DOTATATE (NETSPOT). ${ }^{68} \mathrm{Ga}$-DOTANOC PET$\mathrm{CT}$ was performed in IPF and nonspecific interstitial pneumonia subjects (21). Probe uptake was higher in IPF subjects, consistent with increased somatostatin expression, and occurred in areas of CT-detected fibrosis. In the IPF subjects, PET signal correlated linearly with the amount of fibrosis on CT. However, somatostatin type 2 receptors are also expressed in "proinflammatory" M1 macrophages, which may be present during fibrosis and fibrogenesis (22). ${ }^{68} \mathrm{Ga}$-DOTATATE was recently used in a prospective clinical trial to image atherosclerotic inflammation (22). In excised carotid plaques, SSTR2 gene expression occurred exclusively in M1 macrophages. ${ }^{68} \mathrm{Ga}$-DOTATATE tissue-to-blood ratios correctly identified culprit versus nonculprit arteries in patients with acute coronary syndrome and transient ischemic attack/stroke.

Within the liver, hepatic stellate cells (HSCs) become activated and differentiate into myofibroblasts (23). This activation is essential to the development of hepatic fibrosis (24). Increased expression of the $\alpha_{v} \beta_{3}$ integrin in activated HSCs affects their proliferation and survival $(25,26)$. The binding of $\alpha_{v} \beta_{3}$ to the ECM is dependent on an amino acid complex consisting of arginineglycine-aspartic acid (RGD) (27). SPECT with ${ }^{99 \mathrm{~m}} \mathrm{Tc}$ conjugated to a cyclic RGD peptide was used to assess activated HSCs in a $\mathrm{CCl}_{4}$-induced rodent model of liver fibrosis (28). Increased $\alpha_{\mathrm{v}} \beta_{3}$ expression, determined by an increased liver-to-heart signal ratio, was higher in $\mathrm{CCl}_{4}$-treated rats than in uninjured rats, with the greatest liver-to-heart signal observed in histologically confirmed 
advanced fibrosis. ${ }^{99 \mathrm{~m}} \mathrm{Tc}-3 \mathrm{PRGD} 2$, a probe with two cyclic RGD moieties, and SPECT/CT imaging were used to monitor the development and resolution of thioacetamide-induced liver fibrosis in rats (29). Liver-to-background ratios correlated with the amount of collagen and expression of $\beta_{3}$ integrin. Thioacetamide withdrawal and treatment with IFN- $\alpha 2 \mathrm{~b}$ resulted in a significant decrease of ${ }^{99 \mathrm{~m}} \mathrm{Tc}$-3PRGD2 liver uptake.

RGD-based probes have been used to image myofibroblasts in cardiac tissue after myocardial infarction $(30,31) .{ }^{99 \mathrm{~m}} \mathrm{Tc}$-labeled Cy5.5-RGD imaging peptide ( ${ }^{99 \mathrm{~m} T c-C R I P) ~ S P E C T ~ w a s ~ p e r f o r m e d ~}$ using a mouse model of myocardial infarction resulting from artery occlusion (30). In contrast to healthy mice, tracer uptake was greatest in the infarcted area 2 weeks after myocardial injury and decreased at subsequent measured time points. ${ }^{99 \mathrm{~m} T c-C R I P}$ uptake colocalized to myofibroblasts, which were identified by $\alpha$-smooth muscle actin staining. The degree of ${ }^{99 \mathrm{~m}} \mathrm{Tc}$-CRIP uptake was mitigated in mice treated with an angiotensin-converting enzyme inhibitor and/or an angiotensin II receptor blocker. A similar RGD probe, ${ }^{99 \mathrm{~m}} \mathrm{Tc}-\mathrm{RGD}$ imaging peptide (RIP), was used at weeks 1,3 , and 8 after myocardial infarction in 10 subjects. Myocardial uptake at 3 weeks corresponded to the location of scar at 1 year determined by late-gadolinium enhancement on MRI (32).

\section{Matrix deposition and cross-linking}

Fibrosis results from increased deposition of ECM proteins (33). Activated fibroblasts and myofibroblasts produce structural proteins, including type I collagen, fibronectin, and elastin, which together form fibrous tissue in the area of injury $(33,34)$. Type I collagen is the most abundant collagen in the body (35). Collagen is secreted from the cell as procollagen and undergoes modification, cleavage, and cross-linking to form mature collagen fibrils (36). At present, no available clinical imaging modalities can assess the activity of collagen synthesis or the degree of collagen deposition. Currently used imaging techniques detect the end result of collagen deposition, such as cirrhosis or fibrotic interstitial lung abnormalities.

EP-3533 is a peptide-based gadolinium probe with specificity for type I collagen (37). EP-3533-enhanced MRI was first evaluated in mouse models of myocardial infarction $(37,38)$. Imaging performed at 40 days after myocardial injury demonstrated sustained signal enhancement of the infarcted myocardium, and this signal was demonstrated to be specific to collagen binding (37). The circumferential extent of myocardial scarring was measured in high accuracy with EP-3533-enhanced MRI, using Picrosirius red staining of excised myocardium as the gold standard (38). EP-3533 MRI was also used to assess the amount of type I collagen in liver fibrosis, pulmonary fibrosis, and pancreatic cancer (39-42). In murine models of carbon tetrachloride-induced $\left(\mathrm{CCl}_{4}-\right.$ induced) liver fibrosis and bleomycin-induced pulmonary fibrosis, EP-3533 accurately detected collagen deposition with changes in magnetic resonance (MR) signals correlating linearly to collagen content determined biochemically by hydroxyproline $(40,41)$. Within pancreatic cancer, the accumulation of fibrotic stroma plays an important role, with the extent of stromal growth (desmoplasia) inversely correlating with survival (5). The fibrotic tumor stroma also contributes to the resistance of pancreatic cancer to chemotherapy, making stroma-targeted therapies of great inter- est (43). In a mouse model of pancreatic ductal adenocarcinoma, delayed EP-3533 signal enhancement correlated to the amount of hydroxyproline on histologic analysis (42).

To enable clinical translation of this technology, an improved probe, CM-101, was developed (44). CM-101 uses the more stable macrocyclic gadolinium chelate, Gd-DOTA, and has improved pharmacokinetic properties compared with EP-3533, with decreased blood half-life, and less probe retention in the liver, bone, and kidneys. CM-101-enhanced MRI was performed in two rodent models of liver fibrosis induced by $\mathrm{CCl}_{4}$ or bile duct ligation (BDL), and increased image contrast was observed in the fibrotic animals with signal correlating with hydroxyproline content and morphometric analysis of collagen by histologic analysis.

The same type I collagen-binding peptide was also adapted for PET imaging $(45,46) .{ }^{68} \mathrm{Ga}-\mathrm{CBP} 8$ binds type I collagen with low micromolar affinity and is quickly cleared from the circulation into the urine (45). In two mouse models of bleomycin-induced pulmonary fibrosis, there was an excellent correlation between probe lung uptake and the amount of fibrosis at 7 and 14 days, as determined by Ashcroft score (a numerical index based on microscopy scoring of lung specimens) as well as hydroxyproline content. Probe uptake occurred in areas of fibrosis but not in areas of normal lung. Samples of explanted human lung tissue from pulmonary fibrosis patients undergoing lung transplantation were incubated with ${ }^{68} \mathrm{Ga}-\mathrm{CBP} 8$ to determine the ability of the probe to bind to human tissue. The degree of probe binding correlated positively with collagen content. Together these results suggest that ${ }^{68} \mathrm{Ga}-\mathrm{CBP} 8$ can accurately detect and stage collagen deposition.

Several other molecular probes allow for noninvasive collagen detection using SPECT or PET imaging techniques (45, 47-49). Collagelin is a high-affinity collagen probe identified from a screen focused on the platelet glycoprotein VI binding site on collagens I and III (47). ${ }^{99 \mathrm{~m}} \mathrm{Tc}$-collagelin imaging was performed in mouse models of myocardial infarction and pulmonary fibrosis, with tracer uptake occurring in areas of histologically confirmed fibrosis. Another type I collagen-targeted SPECT probe, ${ }^{99 \mathrm{~m}} \mathrm{Tc}-\mathrm{CBP} 1495$, was used to detect liver and pulmonary fibrosis in rat models, with probe uptake correlating with organ hydroxproline content (49).

Elastin is an ECM protein that is abundant late in the fibrosis process $(50,51)$. A gadolinium-based elastin-specific probe, Gd-ESMA, was reported to detect atherosclerosis extent using apolipoprotein E-deficient mice fed a high-fat diet (HFD) (52). GdESMA demonstrated rapid clearance from the blood pool with low uptake in organs except for the kidneys. Mice were imaged at 4, 8, and 12 weeks after the start of HFD. Vessel wall signal increased as the degree of atherosclerosis progressed and decreased in the setting of statin therapy. Gd-ESMA-enhanced liver imaging was performed in mice treated with $\mathrm{CCl}_{4}$, with increased signal intensity detected in the livers of $\mathrm{CCl}_{4}$-treated versus untreated mice (53).

Collagen cross-linking is a marker of active fibrogenesis and is dependent on ECM-associated enzymes like the lysyl oxidases (36). These enzymes catalyze the oxidation of lysine residues to the aldehyde allysine as a precursor to the formation of crosslinked collagen (54). Gd-Hyd is an allysine-reactive MR probe that detects fibrogenesis (55). In normal mice, Gd-Hyd quickly cleared from the blood with no uptake in the liver or lungs. Gd-Hydenhanced MRI was performed in bleomycin-treated mice, with 
the Gd-Hyd-induced change in lung-to-muscle contrast-to-noise ratio (CNR) being the primary measure. The change in CNR peaked at week 2 and decreased at week 4 , consistent with a spontaneous decrease in active fibrogenesis over time. The time course of the change in CNR paralleled tissue lysyl oxidase activity and total allysine content of the lung.

An oxyamine group substitution for the hydrazide in Gd-Hyd gave Gd-OA (56). Gd-OA binds allysine with a greater affinity than Gd-Hyd and also has a higher relaxivity, a measure of its signal-enhancing capability. Gd-OA had a rapid blood clearance with no uptake in the lungs of healthy mice, but high uptake in the lungs of bleomycin-injured mice, resulting in higher MRI signal. Treatment with a lysyl oxidase inhibitor resulted in decreases in lysyl oxidase activity, total allysine lung content, and MRI signal change to values approaching those of uninjured mice.

\section{Fibrosis-associated processes}

Vascular leak is one of the cardinal responses to tissue injury (13). While necessary to promote resolution of disrupted tissue integrity, marked increases in vascular leak can become pathogenic. The persistence or overexuberance of leak can contribute to the development of fibrosis (57). Gadofosveset is an FDA-approved gadolinium-based contrast agent that reversibly binds serum albumin. After injection, at least $80 \%$ of circulating gadofosveset is albumin-bound at any one time (58). Gadofosveset can image vascular leak by assessing extravascular albumin accumulation. Gadofosveset's ability to detect albumin extravasation was validated using Evans blue dye (EBD) in a mouse model of accelerated atherosclerosis (59). Using apolipoprotein E-deficient mice fed HFD, MR signal intensity of the brachiocephalic artery vessel wall correlated with degree of EBD uptake and increased with atherosclerotic progression (59). We performed gadofosveset-enhanced lung MRI to detect vascular leak in patients with pulmonary fibrosis (60). Using an albumin extravasation index (the change in lung parenchyma signal intensity with contrast administration normalized to the change in aorta signal intensity), we found that the lungs of patients with pulmonary fibrosis had increased albumin extravasation in all measured lung regions compared with the lungs of healthy volunteers. The increased vascular leak detected in pulmonary fibrosis occurred in areas of radiographically normal lung, as determined by CT, in addition to areas of known fibrosis.

Tissue injury also triggers the coagulation cascade as part of the normal wound-healing response, resulting in the formation of extravascular fibrin (61); however, dysregulated coagulation contributes to the development of fibrosis (62). Through protease-activatedreceptor signaling, coagulation factors like thrombin can trigger multiple profibrotic processes, including the influx of profibrotic mediators, TGF- $\beta$ activation, fibroblast proliferation, and myofibroblast differentiation (63). EP-2104R is a fibrin-specific gadolinium-based contrast agent that showed good efficacy in detecting thrombi in preclinical and clinical studies $(64,65)$. EP-2104R binds fibrin with a high specificity and has an 18 -fold higher relaxivity than conventional gadolinium contrast agents (66). EP-2104R-enhanced lung MRI was used to detect increased fibrin deposition associated with fibrosis and to monitor the treatment effect of the thrombin inhibitor dabigatran in a murine model of pulmonary fibrosis (67).
Fibrin deposition in the liver was also evaluated using Gd-P, a peptide-gadolinium probe that binds to the fibrin-fibronectin complex (68), which is increased during liver injury (69). Using a $\mathrm{CCl}_{4}$ model of liver fibrosis, greater signal enhancement was demonstrated in injured mice compared with controls (70).

The innate and the adaptive immune system also become active in response to tissue injury (71), resulting in an influx of mononuclear cells and macrophages to the area of injury and the release of profibrotic cytokines, such as TGF- $\beta$, that activate fibroblasts and promote myofibroblast differentiation (33). Macrophage activity has been assessed using the cysteine cathepsin optical imaging probe BMV109 (72). Dual PET and optical imaging was performed in bleomycin-treated mice using ${ }^{64} \mathrm{Cu}-\mathrm{BMV} 101$. PET signal uptake peaked at day 14 and decreased by day 21 . This decrease at day 21 paralleled a reduction in macrophages detected on immunofluorescence. ${ }^{68} \mathrm{Ga}$-BMV101 PET imaging was performed in a cohort of 3 subjects with IPF, 3 subjects with nonclassifiable pulmonary fibrosis, and 3 healthy controls (72). Standardized uptake values (SUVs) for all three groups were similar in the liver; however, SUVs in the lung were greatest in the IPF group, with nonclassifiable pulmonary fibrosis values closer to those of healthy controls.

Myeloperoxidase (MPO) is released in the setting of neutrophil degranulation and activated macrophages (73). Increased MPO has been implicated in the pathogenesis of multiple disease processes, including atherosclerosis and NASH $(74,75)$. MPO may be important in the development of liver fibrosis through activation of hepatic stellate cells (HSCs) (76). The gadolinium contrast agent MPO-Gd was used to detect MPO activity in early myocardial infarcts in a mouse model of coronary artery ligation (77). MPO-Gd was also able to differentiate steatohepatitis from steatosis in mice and in human liver biopsy specimens (78).

\section{Assessing target expression and confirming target engagement}

There is increasing recognition that heterogeneity within fibrotic diseases, such as IPF, is to some degree the result of underlying molecular heterogeneity yet to be elucidated (79). In oncology, the ability to stratify disease molecularly has revolutionized cancer care by allowing for a personalized and targeted treatment approach. Identifying molecular heterogeneity becomes a central issue for drug development for fibrotic diseases as clinical trials for new therapies may fail when tested on all comers but could prove beneficial when tested in a subgroup with increased target expression. Without the ability to molecularly phenotype such patients, an effective treatment for a subset of patients may not be recognized. As tissue sampling can carry significant risk in fibrotic diseases (80), noninvasive strategies for assessing target expression are needed.

The $\alpha_{\mathrm{v}} \beta_{6}$ integrin is an important activator of the profibrotic cytokine TGF- $\beta$ (81). $\alpha_{\mathrm{v}} \beta_{6}$ is expressed on epithelial cells and upregulated in tissue injury (82). Increased $\alpha_{v} \beta_{6}$ expression has been demonstrated in fibrosis and cancer $(83,84)$. In the bleomycin model, an anti- $\alpha_{v} \beta_{6}$ antibody mitigated the development of fibrosis (85). A phase IIa clinical trial evaluating the safety and efficacy of an $\alpha_{\mathrm{v}} \beta_{6}$ humanized monoclonal antibody in IPF has been completed (ClinicalTrials.gov identifier NCT01371305). A20FMDV2 is a ligand with high affinity and specificity for $\alpha_{v} \beta_{6}$ that has been used to perform noninvasive imaging of $\alpha_{\mathrm{v}} \beta_{6}$ expression $(86,87) .{ }^{111}$ In-labeled 
A2OFMDV2 SPECT/CT imaging detected increased expression of $\alpha_{v} \beta_{6}$ in bleomycin-treated versus untreated mice, and probe uptake was reduced when a large blocking dose of an anti- $\alpha_{v} \beta_{6}$ antibody was administered (87). The degree of expression correlated with the amount of $\alpha_{\mathrm{v}} \beta_{6}$ protein and hydroxyproline content. This probe is being translated into humans with IPF for detection of $\alpha_{v} \beta_{6}$ expression using PET. A clinical trial is under way to evaluate the ability of a fluorine-18-labeled analog, [18F]FP-R01-MG-F2, to detect $\alpha_{\mathrm{v}} \beta_{6}$ in IPF subjects (ClinicalTrials.gov identifier NCT03183570).

Lysophosphatidic acid (LPA) is an important profibrotic mediator that signals though its receptor $\mathrm{LPA}_{1}$ to promote vascular leak and fibroblast recruitment (88). The LPA/LPA pathway contributes to the development of fibrosis across multiple human disease processes (88-92), and a phase II clinical trial of an LPA receptor antagonist in IPF demonstrated a reduction in the degree of pulmonary function decline in the treatment arm (93). ${ }^{11} \mathrm{C}-\mathrm{BMT}-136088$, a PET radioligand for the LPA receptor (94), has been used in rhesus monkeys to determine $\mathrm{LPA}_{1}$ receptor expression in the lungs and other organs, volume of drug distribution, and amount of LPA receptor antagonist needed to block half of the receptor uptake of the PET probe (94).

\section{Diagnosis and staging}

While specific imaging findings can obviate the need for tissue sampling, currently used imaging techniques often detect established or late-stage disease, making noninvasive methods for early diagnosis of fibrosis an urgent need. Staging of certain fibrotic diseases, such as liver fibrosis, still relies on histologic assessment, making a noninvasive staging strategy an ideal clinical alternative. Several of the imaging probes discussed were used early in the time course of murine models of fibrosis. In the bleomycin model, the EP-3533enhanced lung MR signal increased as early as day 5 after bleomycin injury (41), while ${ }^{68} \mathrm{Ga}-\mathrm{CBP} 8$ detected collagen deposition at day 7 (45). The use of EP-3533 in murine models of liver fibrosis was also able to detect early fibrotic changes, e.g., distinguishing Ishak stage 0 from Ishak stage 2 in histologically scored tissue $(39,95)$. EP-3533-enhanced MR was performed alongside MR elastography (MRE) in rats with liver fibrosis induced by different durations of diethylnitrosamine (95). Rats were staged for degree of fibrosis by Ishak score and collagen proportionate area. EP-3533 distinguished early-stage fibrosis with a high sensitivity, whereas MRE was best at distinguishing late-stage fibrosis. Combining EP-3533 molecular imaging with MRE increased the diagnostic accuracy for all stages of disease. Together these data suggest that collagen-specific probes can detect early fibrosis and noninvasively stage disease; however, validation in human fibrotic conditions is needed.

\section{Measuring disease activity and cohort enrichment}

Fibrotic diseases are also clinically heterogeneous in terms of disease progression. In IPF there can be marked differences in pace of disease progression between patients (96). This heterogeneity is also prominent in NASH, as some patients progress to cirrhosis while some experience disease stability or even regression (97). There are no clinically used methods to assess fibrotic disease activity or the pace at which an individual's disease is progressing at any one point in time. From a clinical perspective, the determination of disease activity would enable improved prognostication and tailoring of an individual patient's treatment plan. From a drug development perspective, the ability to determine disease activity would allow clinical trials for evaluation of novel therapies to be enriched with subjects most at risk for disease progression and most likely to benefit should a therapy be effective (98). Such a cohort enrichment strategy may increase statistical power, thereby reducing the number of subjects needed to meet a prespecified endpoint and thereby decrease overall trial costs.

Several molecular imaging targets are aptly suited for assessing disease activity in human fibrotic diseases. As type I collagen is the major ECM protein present in fibrotic tissue, recognition of freshly deposited type I collagen may serve as a marker of disease activity. When evaluated in two models of pulmonary fibrosis, the type I collagen-specific PET probe ${ }^{68} \mathrm{Ga}-\mathrm{CBP} 8$ accurately detected collagen deposition (45). Minimal probe uptake was detected in bone and skin, suggesting that ${ }^{68} \mathrm{Ga}-\mathrm{CBP} 8$ recognizes recently synthesized collagen. A clinical trial is currently ongoing to evaluate the ability of ${ }^{68} \mathrm{Ga}-\mathrm{CBP} 8$ to detect collagen deposition in IPF subjects (ClinicalTrials.gov identifier NCT03535545).

Since collagen cross-linking precedes the development of mature collagen, imaging of lysyl oxidase activity may be used to detect fibrogenesis. Gd-Hyd-enhanced MR accurately detected disease progression caused by $\mathrm{CCl}_{4}$ and reduced fibrogenesis in mice after withdrawal of $\mathrm{CCl}_{4}$ as assessed by histology and lysyl oxidase gene expression (55). These results suggest that lysyl oxidase-targeted MR probes can specifically detect and monitor fibrogenesis. Such information may be of significant clinical benefit as currently used imaging techniques cannot differentiate between new and established fibrosis at any one point in time.

\section{Determining treatment response and developing surrogate endpoints}

Determination of treatment response for fibrotic diseases has relied on histologic changes, organ functional assessments, such as pulmonary function, and mortality. In NASH, for example, assessment of treatment response for investigational therapies has hinged on histologic analysis $(99,100)$. In IPF, recently conducted clinical trials have used change in forced vital capacity at 52 weeks as the primary outcome $(6,7)$. As disease progression within fibrotic diseases may be slow, prolonged trial duration is needed to detect such primary outcomes. Thus, surrogate endpoints for fibrotic diseases are needed to enable earlier determinations of treatment response, thereby reducing trial duration and increasing trial feasibility $(101,102)$. Because of the ability to noninvasively detect upregulated pathways involved in fibrosis pathogenesis, molecular imaging becomes a promising candidate.

Several of the imaging probes discussed measured early changes with treatment in animal models of fibrosis. MRI using the MPO-targeted probe MPO-Gd was performed sequentially in mice 4 and 24 hours after myocardial infarction to assess changes in signal intensity with atorvastatin treatment (77). CNR decreased from 4 to 24 hours in mice treated with atorvastatin compared with untreated mice, in which CNR increased over the same time interval. Myofibroblast-targeted imaging with the RGD probe ${ }^{99 \mathrm{~m}} \mathrm{Tc}$-CRIP was performed to assess anti-remodeling therapy after myocardial infarction in mice (31). ${ }^{99 \mathrm{~m}} \mathrm{Tc}-\mathrm{CRIP}$ imaging was performed 4 weeks after infarction in mice treated with losartan, captopril, spironolactone, 


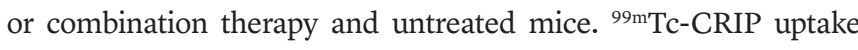
decreased with treatment, with the greatest decrease detected with combination therapy. The degree of ${ }^{99 \mathrm{~m}} \mathrm{Tc}$-CRIP uptake correlated with echocardiographic parameters and histologic measurement of collagen deposition.

PET imaging with the type I collagen-specific probe ${ }^{68} \mathrm{Ga}-\mathrm{CBP} 8$ was performed to assess the effect of an $\alpha_{\mathrm{v}} \beta_{6}$-targeted antibody in the bleomycin model (45). Injured mice receiving antifibrotic treatment had reduced PET signal that paralleled a lower histologic fibrosis score and decreased hydroxyproline content compared with injured mice that received a noneffective antibody. EP-3533 was used to monitor response to rapamycin therapy in a rat BDL model and accurately distinguished therapy responders from nonresponders (103). EP-3533 also quantified reduced fibrosis in BDL rats treated with the farnesoid X receptor agonist EDP-305 (104). In a mouse model of NASH, Gd-Hyd detected reduced fibrogenesis after treatment with EDP-305 (104). Reductions in Gd-Hydenhanced MR signal correlated with decreases in collagen proportionate area, hydroxyproline, and lysyl oxidase gene expression. Gd-Hyd-enhanced MRI could also be combined with other MRI protocols such as quantitative MR fat imaging to provide a comprehensive assessment of NASH. These data suggest that such probes could be used to detect early response to treatment.

\section{Challenges and future directions}

Several important challenges exist in developing molecular imaging probes for human fibrotic disease. First, animal models may not reflect target concentrations in human disease, and probe pharmacokinetics and metabolism may also differ. Furthermore, quantification of sensitivity and specificity will require an accurate truth standard, e.g., histology, which may not be readily available or feasible to obtain. Quantification also requires rigorous assessment of repro- ducibility among subjects and different vendors' scanners. Ultimately outcome data will be required to establish the clinical utility of the imaging test. In addition, imaging probes themselves are novel drugs and require extensive preclinical safety evaluation before they can be tested in humans, although this is relaxed with PET/SPECT probes because of the very low mass doses involved. Despite these challenges, we anticipate that several probes will successfully be translated into clinical use in the next few years (Table 1).

\section{Conclusions}

Over the past decade, molecular imaging has become a valuable tool for the assessment of cardiac disease, neurological disorders, and cancer. It is now poised to address the many pressing and unmet needs in fibrosis, including target engagement, noninvasive assessment of disease stage, patient stratification, and treatment response (Figure 1). While much of the work has relied on preclinical models, the next steps will hinge upon successful translation into humans, and several of the molecular probes available are aptly suited for clinical application. It is our hope that validated molecular probes can be applied to multiple fibrotic indications and aid in the development of novel therapies and the advancement of care for those patients living with these debilitating diseases.

\section{Acknowledgments}

SBM was supported by grants from the Parker B. Francis Foundation and the Scleroderma Foundation. BCF was supported by NIH grants DK104302 and DK104956. PC was supported by NIH grants DK104302, EB009062, HL109448, and HL131907.

Address correspondence to: Peter Caravan, 149 Thirteenth Street, Suite 2301, Charlestown, MA 02129, USA. Phone: 617.643.0193; Email: caravan@nmr.mgh.harvard.edu.
1. Rockey DC, Bell PD, Hill JA. Fibrosis - a common pathway to organ injury and failure. $N$ Engl J Med. 2015;372(12):1138-1149.

2. Wynn TA. Fibrotic disease and the $\mathrm{T}(\mathrm{H}) 1 / \mathrm{T}(\mathrm{H}) 2$ paradigm. Nat Rev Immunol. 2004;4(8):583-594.

3. Burstein B, Nattel S. Atrial fibrosis: mechanisms and clinical relevance in atrial fibrillation. $\mathrm{JAm}$ Coll Cardiol. 2008;51(8):802-809.

4. Rieder F, Fiocchi C. Intestinal fibrosis in inflammatory bowel disease - current knowledge and future perspectives. JCrohns Colitis. 2008;2(4):279-290.

5 . Whatcott CJ, et al. Desmoplasia in primary tumors and metastatic lesions of pancreatic cancer. Clin Cancer Res. 2015;21(15):3561-3568.

6. Richeldi L, et al. Efficacy and safety of nintedanib in idiopathic pulmonary fibrosis. $N$ Engl J Med. 2014;370(22):2071-2082.

7. King TE, et al. A phase 3 trial of pirfenidone in patients with idiopathic pulmonary fibrosis. N Engl J Med. 2014;370(22):2083-2092.

8. Baues M, et al. Fibrosis imaging: current concepts and future directions. Adv Drug Deliv Rev. 2017;121:9-26.

9. Desogere P, Montesi SB, Caravan P. Molecular probes for imaging fibrosis and fibrogenesis [published online ahead of print July 16, 2018]. Chemistry. https://doi.org/10.1002/chem.201801578.

10. Abramson RG, et al. Methods and challenges in quantitative imaging biomarker development. Acad Radiol. 2015;22(1):25-32.

11. Zeisberg M, Kalluri R. Cellular mechanisms of tissue fibrosis. 1. Common and organ-specific mechanisms associated with tissue fibrosis. Am J Physiol Cell Physiol. 2013;304(3):C216-C225.

12. Ahluwalia N, Shea BS, Tager AM. New therapeutic targets in idiopathic pulmonary fibrosis. Aiming to rein in runaway wound-healing responses. Am J Respir Crit Care Med. 2014;190(8):867-878.

13. Singer AJ, Clark RA. Cutaneous wound healing. N Engl J Med. 1999;341(10):738-746.

14. Kendall RT, Feghali-Bostwick CA. Fibroblasts in fibrosis: novel roles and mediators. Front Pharmacol. 2014;5:123.

15. Smith WH, Nair RU, Adamson D, Kearney MT, Ball SG, Balmforth AJ. Somatostatin receptor subtype expression in the human heart: differential expression by myocytes and fibroblasts. J Endocrinol. 2005;187(3):379-386.

16. Gaudillère A, Bernard C, Abello J, Schmitt D, Claudy A, Misery L. Human normal dermal fibroblasts express somatostatin receptors. Exp Dermatol. 1999;8(4):267-273.

17. Borie R, et al. Activation of somatostatin receptors attenuates pulmonary fibrosis. Thorax. 2008;63(3):251-258.

18. Lebtahi R, et al. Increased uptake of 111In-oct- reotide in idiopathic pulmonary fibrosis. $\mathrm{J} \mathrm{Nucl}$ Med. 2006;47(8):1281-1287.

19. Carbone R, et al. Octreoscan perspectives in sarcoidosis and idiopathic interstitial pneumonia. Eur Rev Med Pharmacol Sci. 2003;7(4):97-105.

20. Pettinato C, et al. 68Ga-DOTANOC: biodistribution and dosimetry in patients affected by neuroendocrine tumors. Eur J Nucl Med Mol Imaging. 2008;35(1):72-79.

21. Ambrosini V, et al. 68Ga-DOTANOC PET/CT allows somatostatin receptor imaging in idiopathic pulmonary fibrosis: preliminary results. J Nucl Med. 2010;51(12):1950-1955.

22. Tarkin JM, et al. Detection of atherosclerotic inflammation by $68 \mathrm{Ga}$-DOTATATE PET compared to [18F]FDG PET imaging. J Am Coll Cardiol. 2017;69(14):1774-1791.

23. Tsuchida T, Friedman SL. Mechanisms of hepatic stellate cell activation. Nat Rev Gastroenterol Hepatol. 2017;14(7):397-411.

24. Higashi T, Friedman SL, Hoshida Y. Hepatic stellate cells as key target in liver fibrosis. Adv Drug Deliv Rev. 2017;121:27-42.

25. Li D, He L, Guo H, Chen H, Shan H. Targeting activated hepatic stellate cells (aHSCs) for liver fibrosis imaging. EJNMMI Res. 2015;5(1):71.

26. Zhou X, Murphy FR, Gehdu N, Zhang J, Iredale JP, Benyon RC. Engagement of alphavbeta3 
integrin regulates proliferation and apoptosis of hepatic stellate cells. J Biol Chem. 2004;279(23):23996-24006.

27. Giancotti FG, Ruoslahti E. Integrin signaling. Science. 1999;285(5430):1028-1032.

28. Li F, et al. Molecular imaging of hepatic stellate cell activity by visualization of hepatic integrin av $\beta 3$ expression with SPECT in rat. Hepatology. 2011;54(3):1020-1030.

29. Yu X, et al. Small-animal SPECT/CT of the progression and recovery of rat liver fibrosis by using an integrin $\alpha v \beta 3$-targeting radiotracer. Radiology . 2016;279(2):502-512.

30. van den Borne SW, et al. Molecular imaging of interstitial alterations in remodeling myocardium after myocardial infarction. J Am Coll Cardiol. 2008;52(24):2017-2028.

31. van den Borne SW, et al. Molecular imaging for efficacy of pharmacologic intervention in myocardial remodeling. JACC Cardiovasc Imaging. 2009;2(2):187-198.

32. Verjans J, et al. Early molecular imaging of interstitial changes in patients after myocardial infarction: comparison with delayed contrastenhanced magnetic resonance imaging. J Nucl Cardiol. 2010;17(6):1065-1072.

33. Wynn TA, Ramalingam TR. Mechanisms of fibrosis: therapeutic translation for fibrotic disease. Nat Med. 2012;18(7):1028-1040.

34. Klingberg F, Hinz B, White ES. The myofibroblast matrix: implications for tissue repair and fibrosis. J Pathol. 2013;229(2):298-309.

35. Raghu G, Masta S, Meyers D, Narayanan AS. Collagen synthesis by normal and fibrotic human lung fibroblasts and the effect of transforming growth factor-beta. Am Rev Respir Dis. 1989;140(1):95-100.

36. Mouw JK, Ou G, Weaver VM. Extracellular matrix assembly: a multiscale deconstruction. Nat Rev Mol Cell Biol. 2014;15(12):771-785.

37. Caravan P, et al. Collagen-targeted MRI contrast agent for molecular imaging of fibrosis. Angew Chem Int Ed Engl. 2007;46(43):8171-8173.

38. Helm PA, et al. Postinfarction myocardial scarring in mice: molecular MR imaging with use of a collagen-targeting contrast agent. Radiology. 2008;247(3):788-796.

39. Fuchs BC, et al. Molecular MRI of collagen to diagnose and stage liver fibrosis. J Hepatol. 2013;59(5):992-998.

40. Polasek M, et al. Molecular MR imaging of liver fibrosis: a feasibility study using rat and mouse models. J Hepatol. 2012;57(3):549-555.

41. Caravan P, et al. Molecular magnetic resonance imaging of pulmonary fibrosis in mice. Am J Respir Cell Mol Biol. 2013;49(6):1120-1126.

42. Polasek M, et al. Molecular MR imaging of fibrosis in a mouse model of pancreatic cancer. $S c i$ Rep. 2017;7(1):8114.

43. Erkan M, et al. The role of stroma in pancreatic cancer: diagnostic and therapeutic implications. Nat Rev Gastroenterol Hepatol. 2012;9(8):454-467.

44. Farrar CT, et al. CM-101: type I collagen-targeted MR imaging probe for detection of liver fibrosis. Radiology. 2018;287(2):581-589.

45. Désogère $\mathrm{P}$, et al. Type I collagen-targeted PET probe for pulmonary fibrosis detection and staging in preclinical models. Sci Transl Med. 2017;9(384):eaaf4696.

46. Désogère $\mathrm{P}$, et al. Optimization of a collagen-targeted PET probe for molecular imaging of pulmonary fibrosis. J Nucl Med. 2017;58(12):1991-1996.

47. Muzard J, et al. Non-invasive molecular imaging of fibrosis using a collagen-targeted peptidomimetic of the platelet collagen receptor glycoprotein VI. PLoS One. 2009;4(5):e5585.

48. Velikyan I, et al. Synthesis and preclinical evaluation of 68Ga-labeled collagelin analogs for imaging and quantification of fibrosis. Nucl Med Biol. 2014;41(9):728-736.

49. Zheng $\mathrm{L}$, et al. Molecular imaging of fibrosis using a novel collagen-binding peptide labelled with $99 \mathrm{mTc}$ on SPECT/CT. Amino Acids. 2017;49(1):89-101.

50. Hoff CR, Perkins DR, Davidson JM. Elastin gene expression is upregulated during pulmonary fibrosis. Connect Tissue Res. 1999;40(2):145-153.

51. Blaauboer ME, et al. Novel combination of collagen dynamics analysis and transcriptional profiling reveals fibrosis-relevant genes and pathways. Matrix Biol. 2013;32(7-8):424-431.

52. Makowski MR, et al. Assessment of atherosclerotic plaque burden with an elastin-specific magnetic resonance contrast agent. Nat Med 2011;17(3):383-388.

53. Ehling J, et al. Elastin-based molecular MRI of liver fibrosis. Hepatology. 2013;58(4):1517-1518.

54. Siegel RC. Collagen cross-linking. Synthesis of collagen cross-links in vitro with highly purified lysyl oxidase. J Biol Chem. 1976;251(18):5786-5792.

55. Chen $\mathrm{HH}$, et al. Molecular imaging of oxidized collagen quantifies pulmonary and hepatic fibrogenesis. JCI Insight. 2017;2(11):e91506.

56. Waghorn PA, et al. Molecular magnetic resonance imaging of lung fibrogenesis with an oxyamine-based probe. Angew Chem Int Ed Engl. 2017;56(33):9825-9828.

57. Shea BS, Brooks SF, Fontaine BA, Chun J, Luster AD, Tager AM. Prolonged exposure to sphingosine 1-phosphate receptor-1 agonists exacerbates vascular leak, fibrosis, and mortality after lung injury. Am J Respir Cell Mol Biol. 2010;43(6):662-673.

58. Eldredge HB, Spiller M, Chasse JM, Greenwood MT, Caravan P. Species dependence on plasma protein binding and relaxivity of the gadoliniumbased MRI contrast agent MS-325. Invest Radiol. 2006;41(3):229-243.

59. Phinikaridou A, et al. Noninvasive magnetic resonance imaging evaluation of endothelial permeability in murine atherosclerosis using an albumin-binding contrast agent. Circulation. 2012;126(6):707-719.

60. Montesi SB, et al. Gadofosveset-enhanced lung magnetic resonance imaging to detect ongoing vascular leak in pulmonary fibrosis. Eur Respir J. 2018;51(5):1800171.

61. Furie B, Furie BC. Molecular and cellular biology of blood coagulation. $N$ EnglJMed. 1992;326(12):800-806

62. Scotton CJ, et al. Increased local expression of coagulation factor $\mathrm{X}$ contributes to the fibrotic response in human and murine lung injury. J Clin Invest. 2009;119(9):2550-2563.

63. Mercer PF, Chambers RC. Coagulation and coagulation signalling in fibrosis. Biochim Biophys Acta. 2013;1832(7):1018-1027.
64. Uppal R, Ay I, Dai G, Kim YR, Sorensen AG, Caravan P. Molecular MRI of intracranial thrombus in a rat ischemic stroke model. Stroke. 2010;41(6):1271-1277.

65. Vymazal J, et al. Thrombus imaging with fibrinspecific gadolinium-based MR contrast agent EP-2104R: results of a phase II clinical study of feasibility. Invest Radiol. 2009;44(11):697-704

66. Overoye-Chan K, et al. EP-2104R: a fibrinspecific gadolinium-Based MRI contrast agent for detection of thrombus. J Am Chem Soc. 2008;130(18):6025-6039.

67. Shea BS, et al. Uncoupling of the profibrotic and hemostatic effects of thrombin in lung fibrosis. JCI Insight. 2017;2(9):86608.

68. Ye F, et al. A peptide targeted contrast agent specific to fibrin-fibronectin complexes for cance molecular imaging with MRI. Bioconjug Chem. 2008;19(12):2300-2303.

69. Neubauer K, Knittel T, Armbrust T, Ramadori G. Accumulation and cellular localization of fibrinogen/fibrin during short-term and long-term rat liver injury. Gastroenterology. 1995;108(4):1124-1135.

70. Chow AM, et al. Molecular MRI of liver fibrosis by a peptide-targeted contrast agent in an experimental mouse model. Invest Radiol. 2013;48(1):46-54.

71. Wick G, Backovic A, Rabensteiner E, Plank N, Schwentner C, Sgonc R. The immunology of fibrosis: innate and adaptive responses. Trends Immunol. 2010;31(3):110-119.

72. Withana NP, et al. Non-invasive imaging of idiopathic pulmonary fibrosis using cathepsin protease probes. Sci Rep. 2016;6:19755.

73. Bentwood BJ, Henson PM. The sequential release of granule constitutents from human neutrophils. J Immunol. 1980;124(2):855-862.

74. Zhang R, et al. Association between myeloperoxidase levels and risk of coronary artery disease. JAMA. 2001;286(17):2136-2142.

75. Rensen SS, et al. Increased hepatic myeloperoxidase activity in obese subjects with nonalcoholic steatohepatitis. Am J Pathol. 2009;175(4):1473-1482.

76. Pulli B, et al. Myeloperoxidase-hepatocytestellate cell cross talk promotes hepatocyte injury and fibrosis in experimental nonalcoholic steatohepatitis. Antioxid Redox Signal. 2015;23(16):1255-1269.

77. Nahrendorf M, et al. Activatable magnetic resonance imaging agent reports myeloperoxidase activity in healing infarcts and noninvasively detects the antiinflammatory effects of atorvastatin on ischemia-reperfusion injury. Circulation 2008;117(9):1153-1160.

78. Pulli B, et al. Molecular MR imaging of myeloperoxidase distinguishes steatosis from steatohepatitis in nonalcoholic fatty liver disease. Radiology. 2017;284(2):390-400.

79. Jenkins G. Endotyping idiopathic pulmonary fibrosis should improve outcomes for all patients with progressive fibrotic lung disease. Thorax. 2015;70(1):9-10.

80. Kondoh Y, et al. Acute exacerbation of interstitial pneumonia following surgical lung biopsy. Respir Med.2006;100(10):1753-1759.

81. Sheppard D. Epithelial-mesenchymal interactions in fibrosis and repair. Transforming growth factor- $\beta$ activation by epithelial cells and fibroblasts. 
Ann Am Thorac Soc. 2015;12(suppl 1):S21-S23.

82. Breuss JM, et al. Expression of the beta 6 integrin subunit in development, neoplasia and tissue repair suggests a role in epithelial remodeling. J Cell Sci. 1995;108(pt 6):2241-2251.

83. Henderson NC, Sheppard D. Integrin-mediated regulation of TGF $\beta$ in fibrosis. Biochim Biophys Acta. 2013;1832(7):891-896.

84. Bandyopadhyay A, Raghavan S. Defining the role of integrin alphavbeta6 in cancer. Curr Drug Targets. 2009;10(7):645-652.

85. Horan GS, et al. Partial inhibition of integrin alpha(v)beta6 prevents pulmonary fibrosis without exacerbating inflammation. Am J Respir Crit Care Med. 2008;177(1):56-65.

86. Hausner SH, DiCara D, Marik J, Marshall JF, Sutcliffe JL. Use of a peptide derived from footand-mouth disease virus for the noninvasive imaging of human cancer: generation and evaluation of 4-[18F]fluorobenzoyl A2OFMDV2 for in vivo imaging of integrin alphavbeta 6 expression with positron emission tomography. Cancer Res. 2007;67(16):7833-7840.

87. John AE, et al. Preclinical SPECT/CT imaging of av $\beta 6$ integrins for molecular stratification of idiopathic pulmonary fibrosis. J Nucl Med. 2013;54(12):2146-2152.

88. Tager AM, et al. The lysophosphatidic acid receptor LPA1 links pulmonary fibrosis to lung injury by mediating fibroblast recruitment and vascular leak. Nat Med. 2008;14(1):45-54.

89. Castelino FV, et al. Amelioration of dermal fibrosis by genetic deletion or pharmacologic antagonism of lysophosphatidic acid receptor 1 in a mouse model of scleroderma. Arthritis Rheum. 2011;63(5):1405-1415.

90. Pradère JP, et al. LPA1 receptor activation promotes renal interstitial fibrosis. J Am Soc Nephrol. 2007;18(12):3110-3118.

91. Wu L, et al. Lysophosphatidic acid mediates fibrosis in injured joints by regulating collagen type I biosynthesis. Osteoarthr Cartil. 2015;23(2):308-318.

92. Nakagawa S, et al. Molecular liver cancer prevention in cirrhosis by organ transcriptome analysis and lysophosphatidic acid pathway inhibition. Cancer Cell. 2016;30(6):879-890.

93. Palmer SM, et al. Randomized, double-blind, placebo-controlled, phase 2 trial of BMS986020, a lysophosphatidic acid receptor antagonist for the treatment of idiopathic pulmonary fibrosis. Chest. 2018;154(5):1061-1069.

94. Gallezot JD, et al. Evaluation of the lysophosphatidic acid receptor type 1 radioligand $11 \mathrm{C}-$ BMT-136088 for lung imaging in rhesus monkeys. J Nucl Med. 2018;59(2):327-333.

95. Zhu B, et al. Combined magnetic resonance elastography and collagen molecular magnetic resonance imaging accurately stage liver fibrosis in a rat model. Hepatology. 2017;65(3):1015-1025

96. Ley B, Collard HR, King TE. Clinical course and prediction of survival in idiopathic pulmonary fibrosis. Am J Respir Crit Care Med. 2011;183(4):431-440.

97. Singh S, Allen AM, Wang Z, Prokop LJ, Murad MH, Loomba R. Fibrosis progression in nonalcoholic fatty liver vs nonalcoholic steatohepatitis: a systematic review and meta-analysis of paired-biopsy studies. Clin Gastroenterol Hepatol. 2015;13(4):643-654.e1.

98. Collard HR, et al. A new era in idiopathic pulmonary fibrosis: considerations for future clinical trials. Eur Respir J. 2015;46(1):243-249.

99. Sanyal AJ, et al. Endpoints and clinical trial design for nonalcoholic steatohepatitis. Hepatology. 2011;54(1):344-353.

100. Neuschwander-Tetri BA, et al. Farnesoid X nuclear receptor ligand obeticholic acid for non-cirrhotic, non-alcoholic steatohepatitis (FLINT): a multicentre, randomised, placebo-controlled trial. Lancet. 2015;385(9972):956-965.

101. Raghu G, et al. Idiopathic pulmonary fibrosis: clinically meaningful primary endpoints in phase 3 clinical trials. Am J Respir Crit Care Med. 2012;185(10):1044-1048.

102.Sanyal AJ, Friedman SL, McCullough AJ, Dimick-Santos L, American Association for the Study of Liver Diseases, United States Food Drug Administration. Challenges and opportunities in drug and biomarker development for nonalcoholic steatohepatitis: findings and recommendations from an American Association for the Study of Liver Diseases-U.S. Food and Drug Administration Joint Workshop. Hepatology. 2015;61(4):1392-1405.

103. Farrar CT, et al. 3D molecular MR imaging of liver fibrosis and response to rapamycin therapy in a bile duct ligation rat model. J Hepatol. 2015;63(3):689-696.

104.Erstad DJ, et al. Molecular magnetic resonance imaging accurately measures the antifibrotic effect of EDP-305, a novel farnesoid X receptor agonist. Hepatol Commun. 2018;2(7):821-835. 